\title{
Do pathologists agree on how to diagnose takotsubo cardiomyopathy?
}

\author{
Paolo Angelini ${ }^{1}$
}

Accepted: 15 December 2015/Published online: 28 January 2016

(C) Springer Science+Business Media New York 2016

I read with great interest the report by Indorato et al. [1] regarding takotsubo transient cardiomyopathy (TTC). The authors described a case of sudden death in which the autopsy findings featured apical rupture of the myocardium. In reviewing the details of this case, I began to wonder whether the criteria for diagnosing TTC are well established in the field of pathology. In particular, the authors did not have a pre-mortem electrocardiogram, evidence of apical ballooning (pre- or post-mortem), or an elevation in catecholamine blood levels (the purported etiologic factor) on which to base their diagnosis. Obviously, disappearance of segmental akinesia is the main feature that was absent.

I am particularly interested in this case because I have long been involved in research aimed at elucidating the cause and pathophysiology of this persistently confusing entity [2]. Whenever a patient dies within $24 \mathrm{~h}$ after the onset of TTC-like symptoms, it is clinically important to study the case in detail, as the authors diligently tried to do [1]. Indeed, TTC is a possible explanation for broken-heart syndrome; however, in this case, the following points merit further consideration:

1. The only hint concerning the apical condition is seen in Fig. 3: the apex appears hypertrophic, not dilated, and not thin-walled, as would be typical of acute TTC. Is it possible that in patients with TTC, apical ballooning disappears because of an intracavitary pressure decrease soon after death?

Paolo Angelini

pangelini@leachmancardiology.com

1 Department of Cardiology, Texas Heart Institute, 6624 Fannin, Suite 2780, Houston, TX 77030, USA
2. From a clinical standpoint, I am particularly curious about spasms in this condition [2]; ideally, histologic images of coronary arterial cross-sections would be available. Indeed, some preliminary studies have clearly shown that the only method known to reproduce TTC in recovering patients is acetylcholine testing of endothelial dysfunction [2]. Is it possible to diagnose a spasm after death?

3. A few minutes before the patient died, she had a blood pressure of 122/70 $\mathrm{mmHg}$ and pulse of 95 beats/min. These values do not make a credible case for a catecholamine surge.

4. The histologic findings of eosinophilic infiltration, spotty (not diffuse) coagulation necrosis, and spotty fibrosis could suggest that the pathologic changes were subacute and chronic.

Although TTC is thought to be benign and to have a low mortality rate, a definitive means of diagnosing TTC at autopsy is needed because mortality can occur quite early in this condition, when pathologists may not be ready to establish a diagnosis.

\section{References}

1. Indorato F, Akashi YJ, Rossitto C, Raffino C, Bartoloni G. Takotsubo cardiomyopathy associated with rupture of the left ventricular apex: assessment of histopathological features of a fatal case and literature review. Forensic Sci Med Pathol. 2015;11:577-83.

2. Angelini P. Transient left ventricular apical ballooning: a unifying pathophysiologic theory at the edge of Prinzmetal angina. Catheter Cardiovasc Interv. 2008;71:342-52. 